\title{
A Case of IgG4-Related Disease Presenting as Massive Pleural Effusion and Thrombophlebitis
}

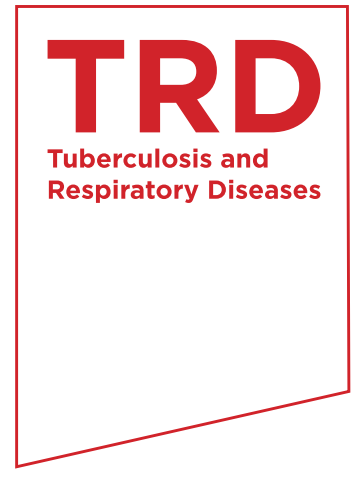

\author{
Jong Hyun Choi, M.D., Jae Kyeom Sim, M.D., Jee Youn Oh, M.D., Eun Joo Lee, M.D., Ph.D., Gyu \\ Young Hur, M.D., Ph.D., Seung Heon Lee, M.D., Ph.D., Sung Yong Lee, M.D., Ph.D., Je Hyeong
} Kim, M.D., Ph.D., Sang Yeub Lee, M.D., Ph.D., Chol Shin, M.D., Ph.D., Jae Jeong Shim, M.D., Ph.D., Kwang Ho In, M.D., Ph.D., Kyung Ho Kang, M.D., Ph.D. and Kyung Hoon Min, M.D., Ph.D. Division of Respiratory and Critical Care Medicine, Department of Internal Medicine, Korea University College of Medicine, Seoul, Korea

Immunoglobulin (Ig) G4-related disease is a recently recognized systemic fibroinflammatory condition characterized by a lymphoplasmacytic infiltrate rich in IgG4-positive plasma cells with elevated circulating levels of IgG4. The disease can either be localized to one or two organs, or present as diffuse multi-organ disease. Furthermore, lesions in different organs can present simultaneously or metachronously. In the pulmonary manefestations, lesions associated with IgG4related disease have been described in the lung parenchyma, airways and pleura, as well as the mediastinum. We report a case of IgG4-related disease presenting as massive pleural effusion and thrombophlebitis.

Keywords: Immunoglobulin G; Pleural Effusion; Thrombophlebitis

\section{Introduction}

Immunoglobulin G4 (IgG4)-related disease is a recently recognized fibroinflammatory condition. It is characterized by lymphoplasmacytic infiltration, storiform fibrosis, phlebitis, abundant IgG4-positive plasma cells and frequently elevated serum IgG4 level. Certain diseases which were thought to be

Address for correspondence: Kyung Hoon Min, M.D., Ph.D.

Division of Respiratory and Critical Care Medicine, Department of Internal Medicine, Korea University Guro Hospital, Korea University College of Medicine, 148 Gurodong-ro, Guro-gu, Seoul 152-703, Korea Phone: 82-2-2626-3308, Fax: 82-2-2626-1166

E-mail: minkyunghoon@korea.ac.kr

Received: Oct. 24, 2013

Revised: Oct. 31, 2013

Accepted: Oct. 31, 2013

(c) It is identical to the Creative Commons Attribution Non-Commercial License (http://creativecommons.org/licenses/by-nc/3.0/).

Copyright (c) 2014

The Korean Academy of Tuberculosis and Respiratory Diseases. All rights reserved. distinct are now accepted as clinical manifestations of individual target organ in IgG4-related disease. IgG4-related disease can involve various organ system ${ }^{1}$. Pulmonary involvement of IgG4-related disease includes airway, lung parenchyma, pleura and mediastinum ${ }^{2}$.

We report a case of IgG4-related disease presenting as massive pleural effusion and thrombophlebitis.

\section{Case Report}

A 48-year-old man was admitted to our hospital complaining of fever, chills, sweating, and dyspnea. He has a history of admission to our hospital for evaluation and management of suggested deep neck infection with small amount of left pleural effusion, which was improved with antibiotic and symptomatic therapy. He had smoked 1 pack per day for 25 years, and taken herbal medication for 1 month before admission, which ameliorated his symptoms a little. He had no underlying illness such as hypertension, diabetes, or autoimmune diseases. On physical examination, he showed generalized edematous appearance with venous engorgement of neck 

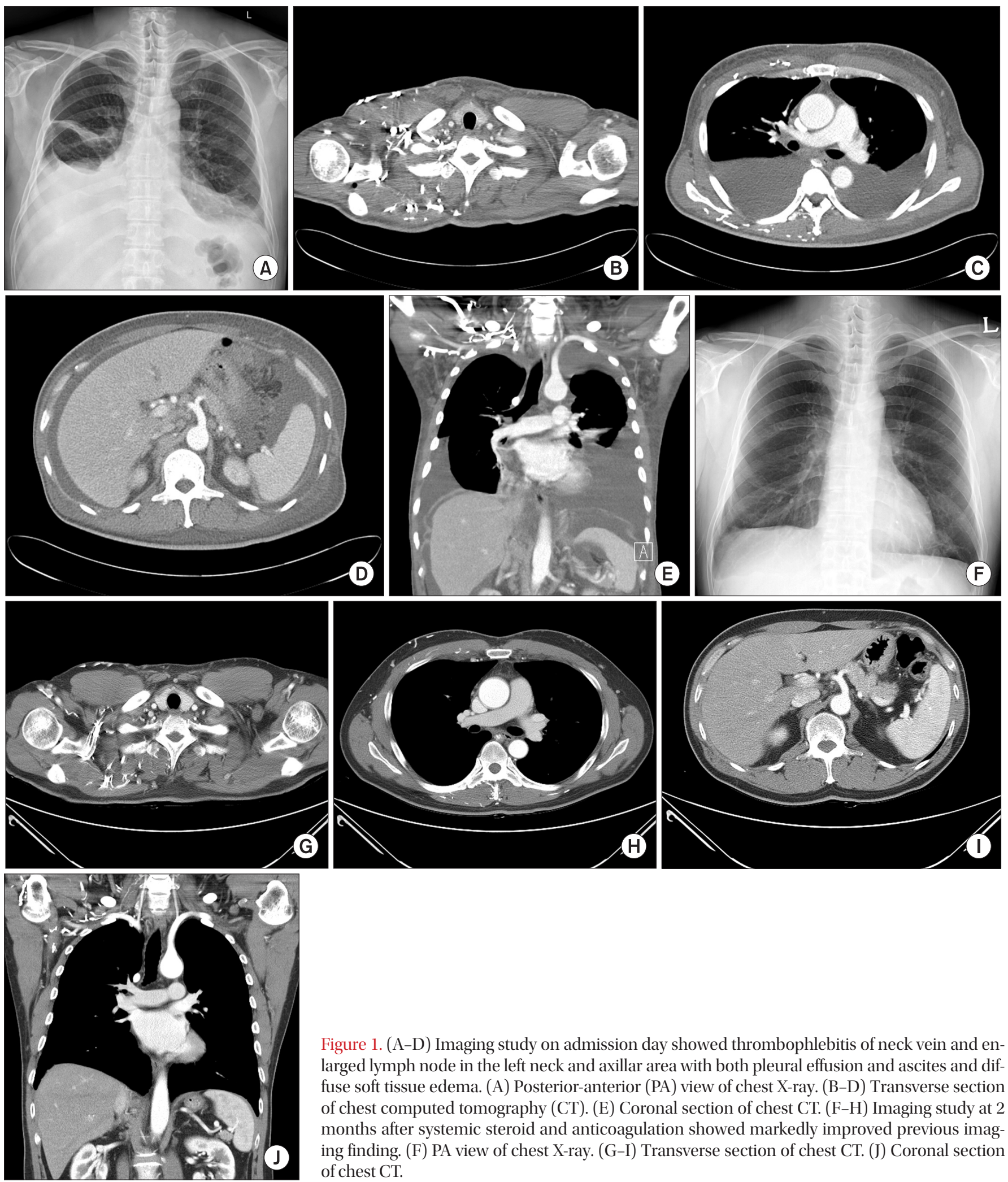

Figure 1. (A-D) Imaging study on admission day showed thrombophlebitis of neck vein and enlarged lymph node in the left neck and axillar area with both pleural effusion and ascites and diffuse soft tissue edema. (A) Posterior-anterior (PA) view of chest X-ray. (B-D) Transverse section of chest computed tomography (CT). (E) Coronal section of chest CT. (F-H) Imaging study at 2 months after systemic steroid and anticoagulation showed markedly improved previous imaging finding. (F) PA view of chest X-ray. (G-I) Transverse section of chest CT. (J) Coronal section of chest CT. 

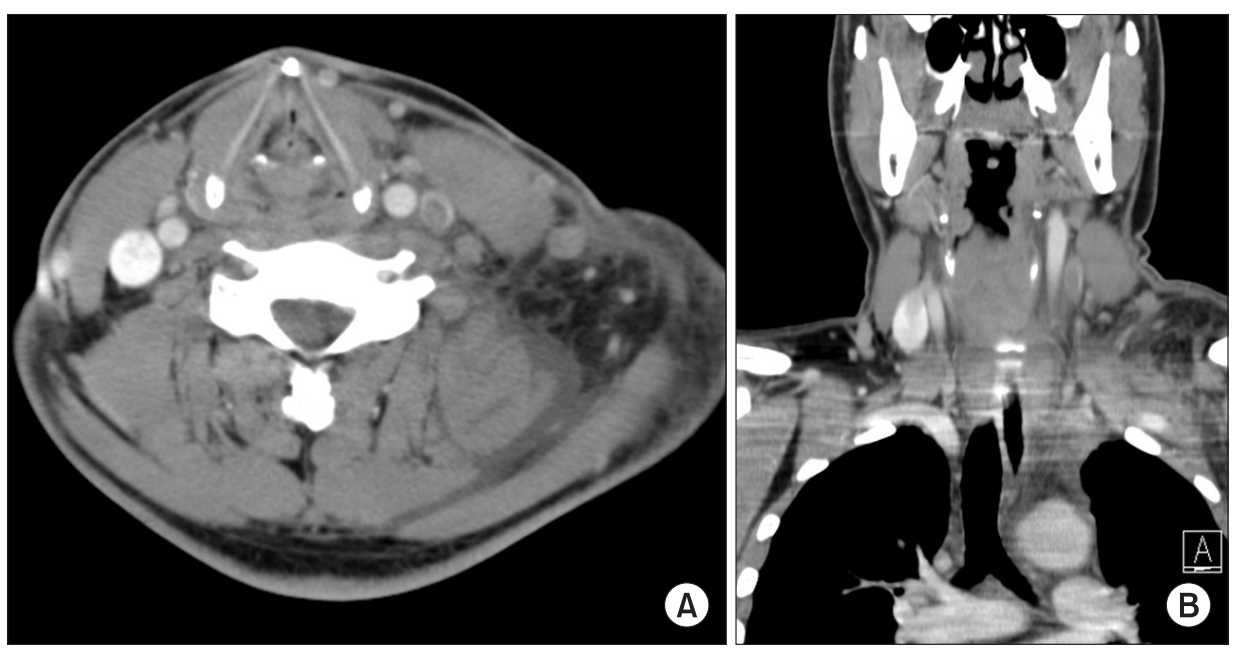

Figure 2. Neck computed tomography on admission day showed showed thrombophlebitis of neck vein and enlarged lymph node at left neck and axillar area. (A) Transverse section. (B) Coronal section.

and upper chest. The lung sound was diminished on both side of lung during auscultation of chest.

Routine laboratory test including liver function test and renal function test showed normal range of results. Autoimmune markers such as fluorescent antinuclear antibody and anti-neutrophil cytoplasmic antibody also showed negative result. Chest X-ray showed massive pleural effusion in both pleural cavity without parenchymal abnormality (Figure 1A). Neck computed tomography (CT) and chest CT showed thrombophlebitis at left innominate vein, subclavian vein and left internal jugular vein and enlarged lymph node at left neck and axillar area with both pleural effusion and ascites and diffuse soft tissue edema (Figures 1B-E, 2). Two-dimentional echocardiography showed spongiform appearance with prominent trabeculation of left ventricle (LV) with preserved LV systolic function, and no significant valvular dysfunction.

Pleural fluid analysis showed lymphocyte predominant exudates nature. But that did not suggest any specific disease. Pleural biopsy through video-assisted thoracoscopic surgery showed chronic inflammation with lymphoplasmacytic infiltration and fibrosis. Plasma cells were positive for IgG and IgG4 in immunohistochemical stain, and the ratio of IgG4 to IgG-positive plasma cells is 0.24 (Figure 3A-E). Lymph node biopsy of neck on previous admission for evaluation and management of suggested deep neck infection also showed same result for IgG and IgG4 stain (Figure 3F-I). Serum IgG4 level was $248.0 \mathrm{mg} / \mathrm{dL}$ (normal range, 6.1-121.4 mg/dL). Laboratory test for common plasma cell disorder showed negative results.

We could diagnose with IgG4-related disease. We started systemic glucocorticoid therapy (prednisolone $0.6 \mathrm{mg} / \mathrm{kg}$ ) with anticoagulation (subcutaneous injection of enoxaparin $1 \mathrm{mg} / \mathrm{kg}$ twice a day). He showed symptomatic improvement after treatment. And the chest X-ray and chest CT which was taken after 2 months of therapy showed almost completely disappeared pleural effusion and ascites, and decreased soft tissue edema (Figure $1 \mathrm{~F}-\mathrm{J}$ ).

\section{Discussion}

Here we reported a man with massive pleural effusion, who was finally found to have IgG4-related disease. Although the concept of IgG4-related disease was accepted recently, it had been introduced much earlier in fact. In 1961, Sarles et al. ${ }^{3}$ described a sclerosing pancreatitis with hypergammaglobulinemia. In 2001, Hamano et al. ${ }^{4}$ revealed the association of high serum IgG4 concentration with sclerosing pancreatitis, and Kamisawa et al. ${ }^{5}$ proposed autoimmune pancreatitis as systemic disease based on histological result of samples from multiple sites which were positive for IgG4.

As in our case, histological confirmation is mandatory for the diagnosis of IgG4-related disease. There are characteristic pathological and immunochemical findings such as lymphoplasmacytic infiltration, storiform fibrosis and obliterative phlebitis, and abundant IgG4-positive plasma cells, respectively. The cellular infiltration contributes to mass formation (tumefactive lesion) and obliteration of vessels. In spite of the existence of these common and well-known features, there are some problems in diagnosing IgG4-related disease. The quantitative assessment of IgG4-positve plasma cell in the tissue is the one. In 2011, Umehara et al. ${ }^{6}$ made comprehensive diagnostic criteria in which IgG4-related disease is classified as definite, probable, possible, and denial. The ratio of IgG4-/ IgG-positive cells in tissue should exceed $40 \%$ and more than 10 IgG4-positive cells per high power field (HPF) should exist on tissue sample ${ }^{6}$. According to the criteria, 'possible' IgG4related disease can be diagnosed to our patient. The consensus statement of the international symposium on IgG4-related disease proposed diagnostic terminology: highly suggestive; probable; insufficient. Our case meets probable diagnostic criteria. Although it requires positive stain for IgG4, it emphasizes pathological features primarily. It even points out the shortness of the ratio of IgG4/IgG in the case of low plasma cell number ${ }^{7}$. In contrast, some only suggested the ratio of IgG4-/ 

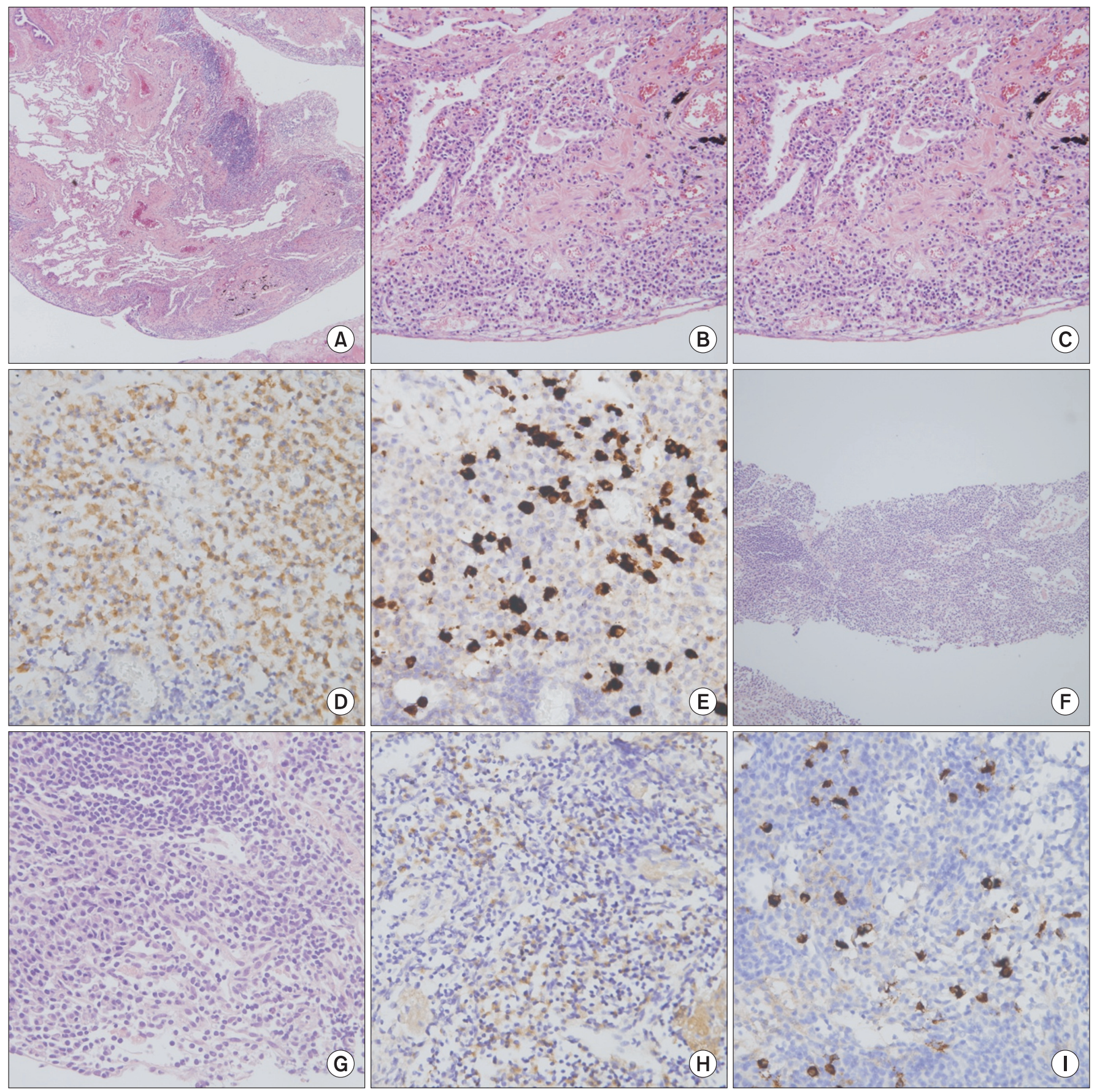

Figure 3. (A-E) Pleural biopsy showed chronic inflammation with lymphoplasmacytic infiltration and fibrosis (A, H\&E stain, $\times 40$; B, H\&E stain, $\times 200$; C, H\&E stain, $\times 400$; D, immunohistochemical stain for IgG, $\times 400$; E, immunohistochemical stain for IgG4, ×400). (F-I) Lymph node biopsy of neck on previous admission also showed chronic inflammation with lymphoplasmacytic infiltration (F, H\&E stain, $\times 40$; G, H\&E stain, $\times 400$; H, immunohistochemical stain for IgG, $\times 400$; I, immunohistochemical stain for IgG4, ×400).

IgG-positive plasma cell $>40 \%$ as a cutoff value for diagnosis of IgG4-related. In a retrospective analysis, they found that as the ratio of IgG4-/IgG-positive plasma cells in tissue increases, the specificity increases and the sensitivity decreases. They also indicated that number of IgG4-positive cells per HPF is di- minished in fibrotic tissue, so it has low specificity ${ }^{8}$. Therefore, more data and validity studies about the diagnostic value and appropriate figure of immunochemical staining are necessary.

There are some interesting findings in our case. First, the pleural effusion is the only pulmonary manifestation. IgG4- 
related lung disease can present in various forms according to involved sites: parenchyma, nodules or masses, or interstitial pneumonia; airway, tracheobroncheal stenosis; pleura, pleural nodules or effusions; mediastinum, lymphadenopathy or fibrosing mediastinitis ${ }^{2}$. The frequency and patterns of involvement are not well established. Zen et al..$^{9}$ collected 21 cases of IgG4-related lung disease, and reported that 5 had pleural lesions. Matsui et al. ${ }^{10}$ analyzed 18 patients retrospectively by their clinical, radiological and pathological features. They found that all 18 patients had hilar and mediastinal lymphadenopathy and nobody had pleural effusion ${ }^{10}$. Although the exact proportion of pleural effusions among IgG4-related lung disease is uncertain, it may be less common manifestation. Second, there is discrepancy between the radiological and pathological findings. In spite of parenchymal involvement on histological examination, chest CT did not show pulmonary infiltrates or mass. The presumed cardiovascular involvement is also noteworthy. His chest CT revealed thrombosis in central veins. Most cases of vascular manifestation deal with arteritis, and cases about venous involvement are uncommon, especially venous thrombosis. A case of IgG4-related middle aged man with left soleal vein thrombus was reported ${ }^{11}$. The spongiform appearance on 2-D echocardiography might be due to cardiac involvement of IgG4-related disease. Until now, reported cases of cardiac involvement include coronary artery (periarteritis, inflammatory aneurysm), pericardium (constrictive pericarditis, pericardial fibrosis), and aorta (periaortitis $)^{12,13}$. Unfortunately, we did not conduct histological confirmation or follow-up echocardiography.

The mainstay of treatment for IgG4-related disease is systemic steroid. Most patients respond well. In the case of autoimmune pancreatitis, highly specific form of IgG4-related disease, steroid responsiveness is one of diagnostic criteria even ${ }^{14}$. Our patient also showed improvement of clinical symptoms and radiological findings after systemic steroid treatment. This responsiveness favors the diagnosis of IgG4related disease in spite of lower ratio of IgG4-/IgG-positive plasma cell. Other therapies, such as rituximab are also proved to be effective in some cases ${ }^{15}$.

IgG4-related disease is not common, but increasingly reported disease entity with systemic involvement. Lung is also a target organ of IgG4-related disease, and manifesting patterns are various. We recommend that clinicians consider IgG4-related disease as a differential diagnostic disease in patient with systemic or localized fibroinflammatory disease with unknown etiology.

\section{Conflicts of Interest}

No potential conflict of interest relevant to this article was reported.

\section{References}

1. Saeki T, Saito A, Hiura T, Yamazaki H, Emura I, Ueno M, et al. Lymphoplasmacytic infiltration of multiple organs with immunoreactivity for IgG4: IgG4-related systemic disease. Intern Med 2006;45:163-7.

2. Ryu JH, Sekiguchi H, Yi ES. Pulmonary manifestations of immunoglobulin G4-related sclerosing disease. Eur Respir J 2012;39:180-6.

3. Sarles H, Sarles JC, Muratore R, Guien C. Chronic inflammatory sclerosis of the pancreas-an autonomous pancreatic disease? Am J Dig Dis 1961;6:688-98.

4. Hamano H, Kawa S, Horiuchi A, Unno H, Furuya N, Akamatsu T, et al. High serum IgG4 concentrations in patients with sclerosing pancreatitis. N Engl J Med 2001;344:732-8.

5. Kamisawa T, Funata N, Hayashi Y, Eishi Y, Koike M, Tsuruta K, et al. A new clinicopathological entity of IgG4-related autoimmune disease. J Gastroenterol 2003;38:982-4.

6. Umehara H, Okazaki K, Masaki Y, Kawano M, Yamamoto M, Saeki T, et al. Comprehensive diagnostic criteria for IgG4-related disease (IgG4-RD), 2011. Mod Rheumatol 2012;22:21-30.

7. Deshpande V, Zen Y, Chan JK, Yi EE, Sato Y, Yoshino T, et al. Consensus statement on the pathology of IgG4-related disease. Mod Pathol 2012;25:1181-92.

8. Masaki Y, Kurose N, Yamamoto M, Takahashi H, Saeki T, Azumi A, et al. Cutoff values of serum IgG4 and histopathological IgG4+ plasma cells for diagnosis of patients with IgG4-related disease. Int J Rheumatol 2012;2012:580814.

9. Zen Y, Inoue D, Kitao A, Onodera M, Abo H, Miyayama S, et al. IgG4-related lung and pleural disease: a clinicopathologic study of 21 cases. Am J Surg Pathol 2009;33:1886-93.

10. Matsui S, Hebisawa A, Sakai F, Yamamoto H, Terasaki Y, Kurihara Y, et al. Immunoglobulin G4-related lung disease: clinicoradiological and pathological features. Respirology 2013;18: 480-7.

11. Kawakami N, Kawai K, Baba N, Ohshima K, Kanekura T. Immunoglobulin G4-positive multi-organ lymphoproliferative syndrome with antiphospholipid antibody syndrome. J Dermatol 2012;39:636-9.

12. Tanigawa J, Daimon M, Murai M, Katsumata T, Tsuji M, Ishizaka N. Immunoglobulin G4-related coronary periarteritis in a patient presenting with myocardial ischemia. Hum Pathol 2012;43:1131-4.

13. Byeon K, Han J, Kim JS, Kim WS, Choe YH, Lee EJ, et al. Immunoglobulin G4-related periaortitis mimicking an intramural hematoma. Ann Thorac Surg 2011;92:1506-8.

14. Chari ST, Smyrk TC, Levy MJ, Topazian MD, Takahashi N, Zhang L, et al. Diagnosis of autoimmune pancreatitis: the Mayo Clinic experience. Clin Gastroenterol Hepatol 2006;4: 1010-6.

15. Khosroshahi A, Carruthers MN, Deshpande V, Unizony S, Bloch DB, Stone JH. Rituximab for the treatment of IgG4-related disease: lessons from 10 consecutive patients. Medicine (Baltimore) 2012;91:57-66. 